\title{
PERSPECTIVE OPEN Immunological surrogate endpoints of COVID-2019 vaccines: the evidence we have versus the evidence we need
}

\author{
Pengfei Jin ${ }^{1}$, Jingxin $\mathrm{Li}^{1,2}$, Hongxing Pan ${ }^{1}$, Yanfei $\mathrm{Wu}^{3}$ and Fengcai $\mathrm{Zhu^{1,2,3 }}$
}

In response to the severe acute respiratory syndrome coronavirus-2 (SARS-CoV-2) pandemic, over 200 vaccine candidates against coronavirus disease 2019 (COVID-2019) are under development and currently moving forward at an unparalleled speed. The availability of surrogate endpoints would help to avoid large-scale filed efficacy trials and facilitate the approval of vaccine candidates, which is crucial to control COVID-19 pandemic. Several phase 3 efficacy trials of COVID-19 vaccine candidates are under way, which provide opportunities for the determination of COVID-19 correlates of protection. In this paper, we review current knowledge for existence of COVID-19 correlates of protection, methods for assessment of immune correlates of protection and issues related to COVID-19 correlates of protection.

Signal Transduction and Targeted Therapy (2021)6:48

; https://doi.org/10.1038/s41392-021-00481-y

\section{INTRODUCTION}

In response to the severe acute respiratory syndrome coronavirus2 (SARS-CoV-2) pandemic, over 200 vaccine candidates against coronavirus disease 2019 (COVID-2019) are under development and currently moving forward at an unparalleled speed. ${ }^{1}$ Among of them, preliminary results from phase 3 efficacy trials are encouraging, with more than $90 \%$ efficacy against COVID-19 diseases for the two mRNA vaccines (BNT162b2 and mRNA-1273), and $70.4 \%$ and $91.4 \%$ efficacy for ChAdOx1 and rAd26/rAd5 COVID-2019 vaccine, respectively. ${ }^{2-5}$ Evaluation of the vaccine efficacy based on efficacy trials capturing clinical disease and/or infection as endpoints is the most direct approach to show the protection of vaccine candidate. ${ }^{6}$ However, phase 3 efficacy trials are very costly and time-consuming, which involve more than thousands of individuals in risk of SARS-CoV-2 exposure in order to provide enough power to show the protective efficacy of vaccine over the placebo. In addition, in the settings of available COVID-19 vaccines approved for emergency use or licensed, it will face a question of ethics to design a randomized placebo-controlled trials for assessing the efficacy of sequent vaccine candidates.

Immune correlate of protection (CoP) is an immunological assay (either humoral or cellular immune response) that reliably predicts protection against disease or infection after vaccination or natural infection. ${ }^{7,8}$ A CoP is of great importance because it can be used as a surrogate endpoint assessing vaccine efficacy without directly observing clinical endpoints. ${ }^{9,10}$ Comparing with large-scale field efficacy trials, immunological trials would save more than $60 \%$ of time and over $80 \%$ of expenses. Table 1 gives the estimates of CoPs for some licensed vaccines, ${ }^{11}$ some of which have been used for the evaluation of vaccine candidates compared with licensed vaccines in immunological trials. The availability of surrogate endpoints help to avoid large-scale filed efficacy trials and facilitate getting vaccine candidates approved, ${ }^{12,13}$ which is crucial to control COVID-19 pandemic. In addition, a CoP could also provide a convenient way to evaluate the immunological level of a population, which is essential in public health strategies.

In this paper, we review current knowledge for existence of COVID-19 correlates of protection, methods to evaluate CoP in efficacy trials from experiences with other vaccines and issues related to COVID-19 correlates of protection.

\section{EVIDENCE FOR EXISTENCE OF COVID-19 CORRELATES OF PROTECTION}

Protective immunity after SARS-CoV-2 natural and experimental infection

The roles of humoral and cellular immune responses being responsible for protective immunity against SARS-CoV-2 in humans have not been fully understood. Most studies available on protective immunity had been performed in animal models. In re-challenge models of rhesus macaques, ${ }^{14,15}$ SARS-CoV-2 infection resulted in the induction of neutralizing antibody (NAb) and cellular immune responses including S-specific $\mathrm{CD}^{+}$and $\mathrm{CD}^{+}$ $T$ cells responses, and provided near-complete protection against SARS-CoV-2 re-challenge. In latter adoptive transfer studies, ${ }^{16}$ it was demonstrated that adoptive transfer of purified IgG from convalescent macaques ${ }^{15}$ protected naïve recipient rhesus macaques against SARS-CoV-2 infection in a dose dependent fashion. In particular, pseudovirus NAb titers of approximately 500 fully protected and titers of approximately 50 partially protected macaques against SARS-CoV-2 infection. In addition, depletion of $\mathrm{CD} 8+\mathrm{T}$ cells in convalescent macaques partially abrogated the protective efficacy against SARS-CoV-2 re-challenge in the setting of NAb titers $<100$, suggesting that cellular immune responses are likely critical for virologic control if NAb titers are near or below the threshold titer required for protection.

\footnotetext{
${ }^{1}$ Department of Vaccine Clinical Evaluation, Jiangsu Province Center for Disease Control and Prevention, Nanjing, China; ${ }^{2} \mathrm{NHC} \mathrm{Key} \mathrm{laboratory} \mathrm{of} \mathrm{Enteric} \mathrm{Pathogenic} \mathrm{Microbiology,}$ Nanjing, China and ${ }^{3}$ Department of Epidemiology and Biostatistics, School of Public Health, Southeast University, Nanjing, China

Correspondence: Fengcai Zhu (jszfc@vip.sina.com)

These authors contributed equally: Pengfei Jin, Jingxin Li
}

Received: 31 December 2020 Accepted: 7 January 2021

Published online: 02 February 2021 


\begin{tabular}{|c|c|c|}
\hline Vaccine & Immune function & Protection level \\
\hline Adenovirus & $\mathrm{Nt} \mathrm{Ab}$ & $1 / 4$ \\
\hline Anthrax & $\begin{array}{l}\text { Toxin Nt Ab, } \\
\text { anti-PA lgG }\end{array}$ & $1 / 3000,10 \mu \mathrm{g} / \mathrm{mL}$ \\
\hline Diphtheria & Toxin Nt Ab & $0.01-0.1 \mathrm{IU} / \mathrm{mL}$ \\
\hline Enterovirus 71 & $\mathrm{Nt} \mathrm{Ab}$ & $1: 16-1: 32$ \\
\hline $\begin{array}{l}\text { H. influenzae } \\
\text { conjugate }\end{array}$ & ELISA Ab & $0.15 \mathrm{ng} / \mathrm{mL}$ \\
\hline Hepatitis A & ELISA Ab & $20 \mathrm{mlU} / \mathrm{mL}$ \\
\hline Hepatitis B & ELISA Ab & $10 \mathrm{mIU} / \mathrm{mL}$ \\
\hline Influenza, inactivated & $\begin{array}{l}\mathrm{HI} \mathrm{Ab} \\
\mathrm{Nt} \mathrm{Ab}\end{array}$ & $\begin{array}{l}1 / 40=50 \% \text { protection, } \\
1 / 320 \text { in children } \\
1 / 40=50 \% \text { protection }\end{array}$ \\
\hline Japanese encephalitis & $\mathrm{Nt} \mathrm{Ab}$ & $1 / 10$ \\
\hline Lyme & ELISA Ab & $1400 \mathrm{U} / \mathrm{mL}$ \\
\hline Malaria & $\begin{array}{l}\text { ELISA Ab } \\
\text { CD4 + T-cell }\end{array}$ & $>10 \mathrm{U} / \mathrm{mL}$ \\
\hline Measles & ELISA Ab & $\geq 120 \mathrm{mlU} / \mathrm{mL}$ \\
\hline Meningococcal & Bactericidal Ab & $\geq 1 / 4$ \\
\hline $\begin{array}{l}\text { Pneumococcal, } \\
\text { conjugated }\end{array}$ & ELISA Ab & $0.35 \mu \mathrm{g} / \mathrm{mL}$ \\
\hline Polio, inactivated/live & $\mathrm{Nt} \mathrm{Ab}$ & $\geq 1 / 8$ \\
\hline Rabies & $\mathrm{Nt} \mathrm{Ab}$ & $\geq 0.5 \mathrm{IU}$ \\
\hline Rubella & ELISA Ab & $\geq 10-15 \mathrm{IU} / \mathrm{mL}$ \\
\hline Smallpox & $\mathrm{Nt} \mathrm{Ab}$ & $\geq 1 / 20-1 / 32$ \\
\hline Tetanus & Toxin Nt Ab & $0.01-0.1 \mathrm{IU} / \mathrm{mL}$ \\
\hline Varicella & GP ELISA & $\geq 5 \mathrm{U} / \mathrm{mL}$ \\
\hline Yellow fever & $\mathrm{Nt} \mathrm{Ab}$ & $\geq 0.7$ LNI \\
\hline
\end{tabular}

$A b$ antibodies, ELISA enzyme-linked immunosorbent assay, GP glycoprotein, $\mathrm{HI}$ hemagglutination inhibition, $L N I$ log neutralization index, Nt neutralization

Since SARS-CoV-2 is a novel pathogen, pre-exiting antibodies are limited. Moreover, it is unknown whether SARS-CoV-2 infection effectively protects against re-exposure in humans. Relatively few studies have been done to assess protective immunity based on observational studies relating pre-exposure immune level to COVID-19 disease or SARS-CoV-2 infection. Encouragingly, the Seattle Boat Study provides the first direct evidence that NAbs protect against SARS-CoV-2 re-infection in humans. ${ }^{17}$ In this outbreak of SARS-CoV-2 on a fishing vessel with an attack rate of $85.2 \%$, none of three crew members who had NAbs (1:174, 1:161 and 1:3082, respectively) prior to departure were infected, whereas 103 of 117 individuals who were seronegative prior to departure were infected. The results implicated NAb as a correlate of protection in humans, but we are unable to speculate a potential protective immunity level form this study due to limited data of pre-exposure NAbs, thus, further studies correlating pre-exposure NAbs with protection are warranted.

SARS-CoV-2-specific memory T cells will likely prove critical for long-term immune protection against COVID-19 and preventing severe COVID-19. Convalescent individuals exhibited robust memory $\mathrm{T}$ cell response months after SARS-CoV-2 infection, even in the absence of detectable specific circulating antibodies against SARS-CoV-2. ${ }^{18,19}$ Pre-existing, cross-reactive T cells have the capacity for accelerating virus clearance with improved clinical outcomes. ${ }^{20}$ Grifoni et al. ${ }^{21}$ identified presence of circulating SARS-CoV-2 specific $\mathrm{CD}^{+} \mathrm{T}$ and $\mathrm{CD}^{+} \mathrm{T}$ cells in most of COVID-19 convalescent patients (70\% and $100 \%$, respectively).
Spike- specific $\mathrm{CD}^{+}{ }^{+} \mathrm{T}$ cell responses correlated well with the magnitude of the anti-spike IgG and IgA titers. Importantly, nonspike-specific $\mathrm{CD}^{+} \mathrm{T}$ cell responses against SARS-CoV-2 were detected in $40-60 \%$ of unexposed individuals, indicating that some potential for cross-reactive induced by common cold coronaviruses. In the subsequent study, ${ }^{22}$ this research team demonstrated a range of preexisting memory $\mathrm{CD} 4+\mathrm{T}$ cells that react to SARS-CoV-2 epitopes actually cross-react with corresponding homologous sequences from many common cold coronaviruses HCoV-OC43, HCoV-229E, HCoV-NL63, or HCoV-HKU1. To identify functional and phenotypic landscape of SARS-CoV-2specific T cell responses across the full spectrum of COVID-19. Sekine et al. $^{19}$ systematically mapped $\mathrm{T}$ cell responses against SARS-CoV-2 in individuals with acute or convalescent COVID-19, exposed family members and unexposed individuals. The results showed that acute-phase SARS-CoV-2-specific T cells displayed a highly activated cytotoxic phenotype that correlated with various clinical markers of disease severity, whereas convalescent-phase SARS-CoV-2-specific T cells were polyfunctional and displayed a stem-like memory phenotype. Importantly, SARS-CoV-2-specific $T$ cells were detectable in antibody-seronegative exposed family members and convalescent individuals with a history of asymptomatic and mild COVID-19. It remains to be determined whether robust memory $T$ cell responses in the absence of detectable circulating antibodies can protect against severe forms of COVID19 in humans. Results from experimental and natural infection studies indicate that SARS-CoV-2 infection can induce protective immunity against reinfection by eliciting NAbs, and cellular immunity is likely critical for virologic control and reducing severity of COVID-19 diseases.

Protective immunity induced by COVID-19 vaccines Most effective human vaccines work by generating antigenspecific functional antibodies, ${ }^{23,24}$ specifically NAbs, which block the entry of the virus into target host cells and prevent infection. Based on the understanding of the interaction of SARS-CoV-2 with host cell, and the immune response to SARS-CoV-2 after nature infection, SARS-CoV-2 Spike protein is identified as an antigenic target for the development of most investigational vaccines. ${ }^{25}$ Hence, the induction of NAbs against SARS-CoV-2 Spike protein is the primary goal of COVID-19 vaccine candidates.

Human challenge trials could accelerate the testing of COVID-19 vaccine candidates and provide rapid assessment for a protective titers of immune CoP by assessing the relationship between antibody titers induced by vaccination and protection postchallenge. ${ }^{26,27}$ Currently, it is controversial due to the severity and unknown long-term impacts of SARS-CoV-2 infection and concerns over ethical administration of such trials. ${ }^{28,29}$ In the absence of data for humans, the result of studies in non-human primates (NHPs) can offer some clues, and help to identify potential CoPs for COVID-19 vaccine candidates. The studies showed that COVID-19 vaccine candidates from different platforms provided protection form infection with SARS-CoV-2 in NHPs, ${ }^{30-36}$ three of which assessed potential immune correlates of protection by taking advantage of the variability in protective efficacy. ${ }^{30,31,35}$ The results showed that vaccine-elicited NAb titers correlated with protection against SARSCoV-2 challenge, suggesting as a potential immune correlate of protection. The findings of DNA and Ad26 vaccine studies in NHPs showed that live virus NAb titers (based on microneutralization assay with $50 \%$ reduction in relative light units as readout) of approximately $100-250\left(10^{2}-10^{2.4}\right)$ were required for complete protection against SARS-CoV-2 infection. ${ }^{30,31}$ Moreover, a logistic regression analysis showed that NAb combined with diverse Fc functional antibody responses improved correlation with protection, which suggests that NAbs have a primary role in protecting against SARS-CoV-2, supported by other binding and functional antibodies (i.e. antibody-dependent complement deposition (ADCD) and antibody-dependent monocyte cellular phagocytosis (ADCP)). 
By contrast, vaccine-elicited ELISPOT responses, and $\mathrm{CD}^{+}$and $\mathrm{CD}^{+}$intracellular cytokine staining responses, did not correlate with protection. These data suggest that NAb may be a potential CoP and NAb titers of 100-250 may be a protective antibody level against SARS-CoV-2 infection in NHPs, although this will need to be confirmed in vaccine efficacy studies in humans.

As we know, there are currently multiple efficacy trials of COVID-19 vaccine candidates under way (see Table 2 ). The results of these COVID-19 vaccine candidates in phase I/II trials have been shown to elicit levels of NAbs being equal to or higher than those observed in convalescent patients, and cellular immune responses. $^{37-46}$ However, the results are difficult to compare due to different assays and readouts were used. Excitingly, the preliminary analysis demonstrated the efficacy of COVID-19 vaccine candidates, ranging from $70 \%$ to $95 \% .^{2-5}$ The promising results will contribute to identify COVID-19 correlates of protection on the basis of data collected from phase III efficacy trials.

\section{METHODS TO EVALUATE COP IN EFFICACY TRIALS FROM EXPERIENCES WITH OTHER VACCINES}

Establishing a cutoff level of immune CoP is the ultimate objective of investigating the immunological CoP. Historically, a variety of study designs and methods have been utilized to evaluate protective immunological level induced by vaccine, which are informative to identifying COVID-19 CoP, including randomized control trials with clinical endpoints, human challenge studies, passive immunization studies, observational studies, the extrapolation of animal studies and so on. Randomized control trials (RCT) provide the ideal context to assess the relationship between vaccine, CoP and clinical endpoints. In a randomized placebocontrolled trial, eligible participants are randomly assigned to receive either vaccine candidate or placebo, followed by collecting blood sample from all participant at the peak of vaccine-induced immune response to assess CoP, with observed clinical endpoints during the surveillance period. Three methods were currently applied to assess a cutoff level of CoP based on numerous vaccine clinical studies, including threshold method, continuous method based on case-cohort study, and receiver operating characteristic (ROC) curve method by using case-control design.

The threshold method for finding the cutoff level of an immunological assay that quantitatively predicts the efficacy of the vaccine was developed in the 7-valent pneumococcal conjugate vaccine efficacy trial. ${ }^{47,48}$ In the threshold method, it assumes a step function between immunological CoP and the probability of disease that individuals with above than the threshold level of CoP are fully protected and blew which individuals remain fully susceptible. The threshold method uses the proportion of vaccinated and unvaccinated individuals below specified thresholds to estimate vaccine efficacy (VE):

$$
\begin{aligned}
\text { VE } & =1-\frac{\operatorname{Pr}[\text { disease }(\text { vaccinated })]}{\operatorname{Pr}[\text { disease }(\text { unvaccinated })]} \\
& =1-\frac{\operatorname{Pr}[\text { individuals less than the cutoff }(\text { vaccinated })]}{\operatorname{Pr}[\text { individuals less than the cutoff }(\text { unvaccinated })]} .
\end{aligned}
$$

In other words, the relative risk of disease equals the relative rate of individuals with immune CoP less than the cutoff, and the predicted vaccine efficacy estimates for various cutoffs were compared with the observed vaccine efficacy. If the predicted vaccine efficacy for a given cutoff is consistent with the observed vaccine efficacy, this cutoff may be the valid cutoff for correlate of protection. Data from three efficacy trials of pneumococcal conjugate vaccines were aggregated to derive a protective threshold of $0.35 \mu \mathrm{g} / \mathrm{mL}$ anti-pneumococcal capsular polysaccharide antibody, which corresponds to the observed VE of $93.0 \%{ }^{49}$ The threshold concentration of $0.35 \mu \mathrm{g} / \mathrm{mL}$ has been recommended by the WHO Working Group as applicable on a global basis for assessing the efficacy of future pneumococcal conjugate vaccines, without the need for further large-scaled efficacy trials.

A limitation of the threshold method is that the relationship between assay values and the occurrence of disease below the threshold level is usually not specified, and individuals with low assay values might not develop disease because of not being exposed. ${ }^{50}$ In addition, individuals with assay values above the chosen threshold will occasionally develop disease. ${ }^{49}$ Theoretically, the relationship between risk of disease and immune response marker can be modeled as a continuous function. To address this problem, Dunning ${ }^{50}$ proposed the scaled logit model for calculating titerspecific rates of disease, which includes the continuous relationship between levels of the immune marker and protection in which exposure is modeled explicitly. In phase III efficacy trials of influenza and enterovirus 71vaccines, the continuous method has been applied to assess respective CoP. ${ }^{51-54}$. The risk of disease could be highly influenced by the probability of exposure to an infectious pathogen. Currently, $\mathrm{HI} \geq 40$ corresponding to $50 \%$ protection from infection was recommended as surrogate of protection to evaluate the efficacy of new influenza vaccines. However, it needs higher $\mathrm{HI}$ titer to provide $50 \%$ protection against influenza illness in children and older individuals. ${ }^{51,52}$ Therefore, it is necessary to appropriately improve the protection level in high-risk population (i.e., old individuals and those with underlying health conditions in COVID19 pandemic) or high prevalence areas. Meanwhile, targeting a high level of protection against the risk of infection can reduce pathogen circulation in population and provide the indirect effects, which has important public health implications.

In the efficacy trials of influenza and enterovirus 71 vaccines, receiver operating characteristic (ROC) curve was also applied to determine the cutoff level of immunological CoP based on casecontrol designs. ${ }^{55-58}$ In the ROC curve, sensitivity was defined as the proportion of individuals with a post-vaccination titer below the cutoff value among those with confirmed clinical disease; specificity was defined as the proportion of individuals with a post-vaccination titer equal to or greater than the cutoff value among those without confirmed clinical disease. The best cutoff value for the immunological CoP could best separates the subjects who were protected from those who were not, at which the sum of the specificity and sensitivity is maximum. In the ROC curve analysis, the Youden index gives the same weight to both sensitivity and specificity, which separates the protected and non-protected individuals. However, the rates of infectious disease among individuals with low titers may be strongly associated with the chance of exposure and disease prevalence, and individuals who were not infected are a mixture of individuals who were protected and those who were unprotected but also unexposed. Therefore, false negatives are likely to occur and sensitivity should determine the cut-off value, and it is necessary to improve sensitivity to reduce false negatives in accordance with the exposure level.

Three methods with different interpretations have been developed to explore the surrogate protection for vaccine clinical trials in terms of absolute and relative $\mathrm{CoP}^{9,11}$ we need to select appropriate methods according to the characteristic of vaccine candidates, different study designs and data collected in ongoing phase III efficacy trials. Therefore, using a CoP in the evaluation of vaccine clinical trials, we should take consideration of other issues related to immunological $\mathrm{CoP}$, including the definition of clinical endpoint, type of vaccine candidates, the target population, exposure intensity, as well as the generalizability of the results to other settings.

\section{OPPORTUNITIES AND CHALLENGES FOR COVID-19 CORRELATES OF PROTECTION}

Over 200 COVID-19 vaccine candidates, based on several different platforms, are currently is moving forward at an unparalleled 


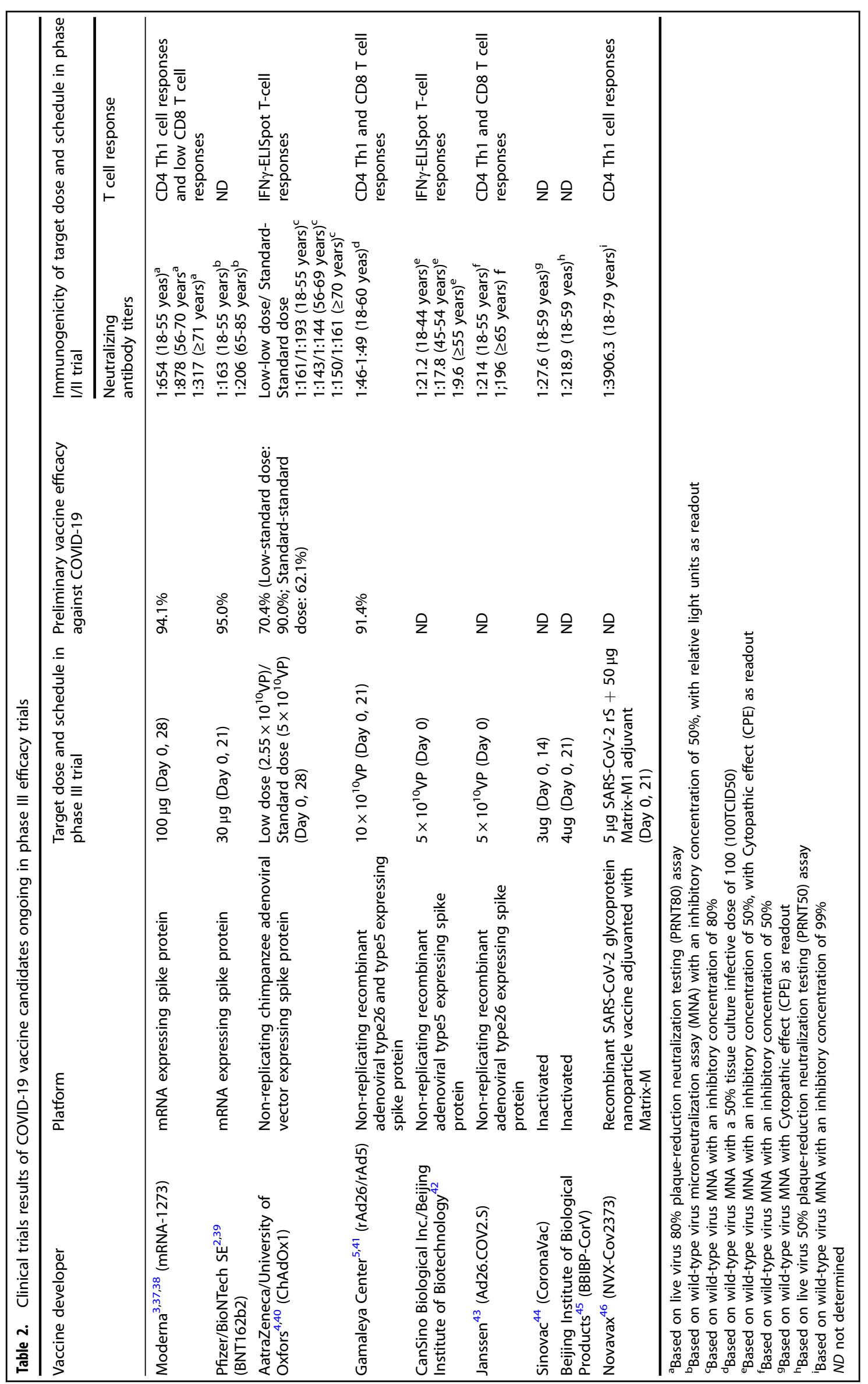


speed, which will provide opportunities to identify COVID-19 correlates of protection. Meanwhile, breadth of global response presents challenges to identification of correlates of protection. It is essential to specify standardized, quantifiable endpoints against which the vaccine is expected to protect, since it may be variable the relationship between CoP and different endpoints. An efficacious COVID-19 vaccine could reduce the likelihood of symptomatic infection, severity of disease, or degree of transmission. In general, NAb and T cellular immunity are needed to protect against severe disease, and mucosal immune response and higher NAb titers could be required to prevent transmission of pathogens. $^{9}$

Notably, vaccine-induced protective immune response may be various for vaccine candidates from different platforms. Cellular immune responses have been described in response to infection and are likely to be an important component of a protective immune response. Among COVID-19 vaccine candidates onging in phase III efficacy trials, vectors vaccines, mRNA vaccines and recombinant protein vaccines are able to elicit T-cell responses, ${ }^{59}$ but it is not clear whether the relative contribution to efficacy of Tcell responses is consistent for different vaccine candidates, which may have an effect on the application of a universal CoP.

NHPs studies provided evidence for antibody-mediated protection against SARS-CoV-2 infection, in which timepoint of challenge is at day 14 or 28 post-vaccination (at the peak point of antibody production), so a key question concerns the durability of antibody responses induced by COVID-19 vaccines. Currently, the immune persistence of COVID-19 vaccines is yet to be known. Several recent studies investigated the durability of the antibody responses (binding antibody and neutralizing antibody) in SARSCoV-2 infection, but opposite results were presented. ${ }^{60}$ As we known, antibody titers pre-exposure is most correlated with disease and infection. NAb titers correlating with clinical endpoints, obtained from clinical trials, may not represent true protective immunity level if there is a "rapid decay" of NAb titers after vaccination.

Measurement of vaccine-induced immune markers is a key component for establishing COVID-19 CoP. A variety of serological assays have been developed, including live virus neutralizing assays in different formats, pseudo virus neutralizing assays using different agents and ELISA assays using different target binding antigens. Comparing immune response against different vaccine candidates is challenging due to lack of standardization among different assays and different testing laboratories. WHO Collaborating Center for Biological Standardization and National Institute for Biological Standards and Control (NIBSC) in UK are developing the WHO international standard and reference panel for antiSARS-CoV-2 antibody. ${ }^{61}$

Efficacy trials have the advantage of identifying the association vaccination, immune markers and protection. However, the enormous resources required to conduct such studies may limit the number that can be done, including the actual number of diseases, the number, quality of biological samples collected, and the number of collection time points. ${ }^{62}$ For example, to determine the immune correlate of protection for inactivated enterovirus 71 vaccine, blood samples from participants of approximately 20,000 at day (before the first dose) and day 56 (28 days after second dose) were collected in two efficacy trials. ${ }^{57,58}$ Differences between vaccines, immune responses and clinical endpoints, and the standardization of assessing immune markers as well as resource needs, present challenges for COVID-19 CoP, so much work remains to be done.

\section{CONCLUDING REMARKS AND PERSPECTIVES}

In conclusion, current NHPs studies indicate that NAb targeting spike protein is a potential and practical CoP supported by other Fc functional antibody responses, and cellular immunity is likely critical for virologic control and reducing severity of COVID-19 diseases. NHPs challenge studies showed that live virus NAb titers of 100-250, based on microneutralization assay with $50 \%$ reduction in relative light units as readout, may be a protective immune level against SARS-CoV-2 infection in NHPs. However, there may be important differences between SARS-CoV-2 infection in macaques and human beings, including racial differences, intensity and timepoint of exposure and other unknown factors, so protective immune level obtained in animal studies would need validation in clinical trials.

With the development of international standards assessing immune markers and the cooperation of international multicenter clinical trials, it will provide opportunities for the determination of COVID-19 correlates of protection.

\section{ACKNOWLEDGEMENTS}

This work was supported by Jiangsu Province Special Funds for Key Research \& Development (2060499).

\section{ADDITIONAL INFORMATION}

Competing interests: The authors declare no competing interests.

\section{REFERENCES}

1. World Health Organization. Draft landscape of COVID-19 candidate vaccines. https://www.who.int/publications/m/item/draft-landscape-of-covid-19candidate-vaccines (2020)

2. Polack, F. P. et al. Safety and efficacy of the BNT162b2 mRNA Covid-19 vaccine. $N$. Engl. J. Med. 383, 2603-2615 (2020).

3. Baden L. R. et al. Efficacy and safety of the mRNA-1273 SARS-CoV-2 vaccine. $N$. Engl. J. Med. https://doi.org/10.1056/NEJMoa2035389 (2020).

4. Voysey, M. et al. Safety and efficacy of the ChAdOx1 nCoV-19 vaccine (AZD1222) against SARS-CoV-2: an interim analysis of four randomised controlled trials in Brazil, South Africa, and the UK. Lancet. https://doi.org/10.1016/S0140-6736(20) 32661-1 (2020)

5. Gamaleya National Center. The Sputnik V vaccine's efficacy is confirmed at $91.4 \%$ based on data analysis of the final control point of clincial trials. https:// sputnikvaccine.com/newsroom/pressreleases/the-sputnik-v-vaccine-s-efficacy-isconfirmed-at-91-4-based-on-data-analysis-of-the-final-control-po/ (2020).

6. Food and Drug Adimistration. Development and Licensure of Vaccines to Prevent COVID-19 Guidance for Industry. https://www.fda.gov/media/139638/download (2020).

7. Plotkin, S. A. \& Gilbert, P. B. Nomenclature for immune correlates of protection after vaccination. Clin. Infect. Dis. 54, 1615-1617 (2012).

8. Qin, L., Gilbert, P. B., Corey, L., McElrath, M. J. \& Self, S. G. A framework for assessing immunological correlates of protection in vaccine trials. J. Infect. Dis. 196, 1304-1312 (2007).

9. World Health Organization. Correlates of vaccine-induced protection: methods and implications. https://apps.who.int/iris/handle/10665/84288 (2013).

10. Hodgson, S. H. et al. What defines an efficacious COVID-19 vaccine? A review of the challenges assessing the clinical efficacy of vaccines against SARS-CoV-2. Lancet Infect. Dis. https://doi.org/10.1016/S1473-3099(20)30773-8 (2020)

11. Plotkin, S. A., Orenstein W. A., Offit, P. A., Edwards, K. M. in Plotkin's Vaccines (7th edition), Correlates of Protection (ed Plotkin, S. A. \& Gilbert, P.) Ch.3, 35 (Elsevier Press, 2018).

12. Sadoff, J. C. \& Wittes, J. Correlates, surrogates, and vaccines. J. Infect. Dis. 196, 1279-1281 (2007).

13. van Els, C. et al. Fast vaccine design and development based on correlates of protection (COPs). Hum. Vaccin. Immunother. 10, 1935-1948 (2014).

14. Deng, W. et al. Primary exposure to SARS-CoV-2 protects against reinfection in rhesus macaques. Science 369, 818-823 (2020).

15. Chandrashekar, A. et al. SARS-CoV-2 infection protects against rechallenge in rhesus macaques. Science 369, 812-817 (2020).

16. McMahan, $\mathrm{K}$. et al. Correlates of protection against SARS-CoV-2 in rhesus macaques. Nature. https://doi.org/10.1038/s41586-020-03041-6 (2020).

17. Addetia, A. et al. Neutralizing antibodies correlate with protection from SARSCoV-2 in humans during a fishery vessel outbreak with a high attack rate. J. Clin. Microbiol. 58, e02107-e02120 (2020).

18. Vabret, N. et al. Immunology of COVID-19: current state of the science. Immunity 52, 910-941 (2020) 
19. Sekine, T. et al. Robust T cell immunity in convalescent individuals with asymptomatic or mild COVID-19. Cell 183, 158-168.e114 (2020).

20. Auladell, M. et al. Recalling the future: immunological memory toward unpredictable Influenza viruses. Front. Immunol. 10, 1400 (2019).

21. Grifoni, A. et al. Targets of T cell responses to SARS-CoV-2 coronavirus in humans with COVID-19 disease and unexposed individuals. Cell 181, 1489-1501.e1415 (2020).

22. Mateus, J. et al. Selective and cross-reactive SARS-CoV-2 T cell epitopes in unexposed humans. Science 370, 89-94 (2020).

23. Plotkin, S. A. Vaccines: correlates of vaccine-induced immunity. Clin. Infect. Dis. 47, 401-409 (2008).

24. Plotkin, S. A. Correlates of protection induced by vaccination. Clin. Vaccin. Immunol. 17, 1055-1065 (2010).

25. Krammer, F. SARS-CoV-2 vaccines in development. Nature 586, 516-527 (2020).

26. Eyal, N., Lipsitch, M. \& Smith, P. G. Human challenge studies to accelerate coronavirus vaccine licensure. J. Infect. Dis. 221, 1752-1756 (2020).

27. Nguyen, L. C. et al. Evaluating use cases for human challenge trials in accelerating SARS-CoV-2 vaccine development. Clin. Infect. Dis. https://doi.org/10.1093/cid/ ciaa935 (2020).

28. Spinola, S. M., Zimet, G. D., Ott, M. A. \& Katz, B. P. Human challenge studies are unlikely to accelerate coronavirus vaccine licensure due to ethical and practical issues. J. Infect. Dis. 222, 1572-1574 (2020).

29. Shah, S. K. et al. Ethics of controlled human infection to address COVID-19. Science 368, 832-834 (2020).

30. $\mathrm{Yu}$, J. et al. DNA vaccine protection against SARS-CoV-2 in rhesus macaques. Science 369, 806-811 (2020)

31. Mercado, N. B. et al. Single-shot Ad26 vaccine protects against SARS-CoV-2 in rhesus macaques. Nature 586, 583-588 (2020).

32. Gao, Q. et al. Development of an inactivated vaccine candidate for SARS-CoV-2. Science 369, 77-81 (2020).

33. Wang, H. et al. Development of an inactivated vaccine candidate, BBIBP-CorV, with potent protection against SARS-CoV-2. Cell 182, 713-721.e719 (2020).

34. van Doremalen, N. et al. ChAdOx $1 \mathrm{nCoV}-19$ vaccine prevents SARS-CoV-2 pneumonia in rhesus macaques. Nature 586, 578-582 (2020).

35. Corbett, K. S. et al. Evaluation of the mRNA-1273 vaccine against SARS-CoV-2 in nonhuman primates. N. Engl. J. Med. 383, 1544-1555 (2020).

36. Guebre-Xabier, M. et al. NVX-CoV2373 vaccine protects cynomolgus macaque upper and lower airways against SARS-CoV-2 challenge. Vaccine 38, 7892-7896 (2020).

37. Jackson, L. A. et al. An mRNA vaccine against SARS-CoV-2 - preliminary report. $N$. Engl. J. Med. 383, 1920-1931 (2020).

38. Anderson, E. J. et al. Safety and immunogenicity of SARS-CoV-2 mRNA-1273 vaccine in older adults. N. Engl. J. Med. 383, 2427-2438 (2020).

39. Walsh, E. E. et al. Safety and immunogenicity of two RNA-based covid-19 vaccine candidates. N. Engl. J. Med. 383, 2439-2450 (2020).

40. Ramasamy, M. N. et al. Safety and immunogenicity of ChAdOx1 $\mathrm{nCoV}-19$ vaccine administered in a prime-boost regimen in young and old adults (COV002): a single-blind, randomised, controlled, phase 2/3 trial. Lancet 396, 1979-1993 (2020).

41. Logunov, D. Y. et al. Safety and immunogenicity of an rAd26 and rAd5 vectorbased heterologous prime-boost COVID-19 vaccine in two formulations: two open, non-randomised phase 1/2 studies from Russia. Lancet 396, 887-897 (2020).

42. Zhu, F. C. et al. Immunogenicity and safety of a recombinant adenovirus type-5vectored COVID-19 vaccine in healthy adults aged 18 years or older: a randomised, double-blind, placebo-controlled, phase 2 trial. Lancet 396, 479-488 (2020).

43. Sadoff, J. et al. Interim Results of a Phase 1-2a Trial of Ad26.COV2.S Covid-19 Vaccine. N. Engl. J. Med. https://doi.org/10.1056/NEJMoa2034201 (2021).

44. Zhang, Y. et al. Safety, tolerability, and immunogenicity of an inactivated SARSCoV-2 vaccine in healthy adults aged 18-59 years: a randomised, double-blind, placebo-controlled, phase $1 / 2$ clinical trial. Lancet Infect. Dis. https://doi.org/ 10.1016/S1473-3099(20)30843-4 (2020).
45. Xia, S. et al. Safety and immunogenicity of an inactivated SARS-CoV-2 vaccine, BBIBP-CorV: a randomised, double-blind, placebo-controlled, phase 1/2 trial. Lancet Infect. Dis. 21, 39-51 (2021).

46. Keech, C. et al. Phase 1-2 trial of a SARS-CoV-2 recombinant spike protein nanoparticle vaccine. N. Engl. J. Med. 383, 2320-2332 (2020).

47. Jodar, L. et al. Serological criteria for evaluation and licensure of new pneumococcal conjugate vaccine formulations for use in infants. Vaccine 21, 3265-3272 (2003).

48. Siber, G. R. et al. Estimating the protective concentration of anti-pneumococcal capsular polysaccharide antibodies. Vaccine 25, 3816-3826 (2007).

49. Angel, J., Steele, A. D. \& Franco, M. A. Correlates of protection for rotavirus vaccines: possible alternative trial endpoints, opportunities, and challenges. Hum. Vaccin. Immunother. 10, 3659-3671 (2014).

50. Dunning, A. J. A model for immunological correlates of protection. Stat. Med. 25, 1485-1497 (2006).

51. Black, S. et al. Hemagglutination inhibition antibody titers as a correlate of protection for inactivated influenza vaccines in children. Pediatr. Infect. Dis. 30 , 1081-1085 (2011)

52. Dunning, A. J. et al. Correlates of protection against influenza in the elderly: results from an Influenza Vaccine Efficacy Trial. Clin. Vaccin. Immunol. 23, 228-235 (2016).

53. Jin, P. et al. Validation and evaluation of serological correlates of protection for inactivated enterovirus 71 vaccine in children aged 6-35 months. Hum. Vaccin. Immunother. 12, 916-921 (2016).

54. Zhu, W., Jin, P., Li, J. X., Zhu, F. C. \& Liu, P. Correlates of protection for inactivated enterovirus 71 vaccine: the analysis of immunological surrogate endpoints. Expert. Rev. Vaccines 16, 945-949 (2017).

55. Barrett, P. N. et al. Efficacy, safety, and immunogenicity of a Vero-cell-culturederived trivalent influenza vaccine: a multicentre, double-blind, randomised, placebo-controlled trial. Lancet 377, 751-759 (2011).

56. Benoit, A. et al. Hemagglutination inhibition antibody titers as a correlate of protection against seasonal A/H3N2 influenza disease. Open Forum Infect. Dis. 2, ofv067 (2015)

57. Zhu, F. C. et al. Efficacy, safety, and immunology of an inactivated alum-adjuvant enterovirus 71 vaccine in children in China: a multicentre, randomised, doubleblind, placebo-controlled, phase 3 trial. Lancet 381, 2024-2032 (2013).

58. Zhu, F. et al. Efficacy, safety, and immunogenicity of an enterovirus 71 vaccine in China. N. Engl. J. Med. 370, 818-828 (2014).

59. DiPiazza, A. T., Graham, B. S. \& Ruckwardt, T. J. T cell immunity to SARS-CoV-2 following natural infection and vaccination. Biochem. Biophys. Res. Commun. https://doi.org/10.1016/j.bbrc.2020.10.060 (2020).

60. Grigoryan, L. \& Pulendran, B. The immunology of SARS-CoV-2 infections and vaccines. Semin. Immunol. 50, 101422 (2020).

61. World Health Organization. Standardization of vaccines for coronavirus disease (COVID-19). https://www.who.int/biologicals/Standardization_Covid-19 (2020).

62. Srikiatkhachorn, A. \& Yoon, I. K. Immune correlates for dengue vaccine development. Expert. Rev. Vaccines 15, 455-465 (2016).

Open Access This article is licensed under a Creative Commons Attribution 4.0 International License, which permits use, sharing, adaptation, distribution and reproduction in any medium or format, as long as you give appropriate credit to the original author(s) and the source, provide a link to the Creative Commons license, and indicate if changes were made. The images or other third party material in this article are included in the article's Creative Commons license, unless indicated otherwise in a credit line to the material. If material is not included in the article's Creative Commons license and your intended use is not permitted by statutory regulation or exceeds the permitted use, you will need to obtain permission directly from the copyright holder. To view a copy of this license, visit http://creativecommons. org/licenses/by/4.0/.

(c) The Author(s) 2021 